\title{
O papel dos sistemas visual, vestibular, somatosensorial e auditivo para o controle postural
}

\author{
The role of visual, vestibular, somatosensory and auditory systems for the postural control
}

\author{
Ana Francisca Rozin Kleiner', Diana Xavier De Camargo Schlittler', \\ Mónica Del Rosário Sánchez-Arias ${ }^{3}$
}

\section{RESUMO}

A manutenção de uma determinada orientação corporal é obtida a partir do complexo relacionamento entre informação sensorial e atividade muscular. Desta forma, o objetivo deste estudo foi revisar o papel das informaçôes visuais, somatossensoriais, vestibulares e auditivas para manutenção e controle postural. Método. foi realizada uma busca nas bases de dados CAPES e PubMed, nos últimos 24 anos, com as seguintes palavras-chave: postural control, sensory information, vestibular system, visual system, somatosensory system, auditory system e haptic system. Resultados. foram analisadas a influência de cada sistema sensorial, bem como sua integração para a manutenção e controle postural. Conclusáo. a literatura apontou que existe uma redundância nas informaçóes fornecidas pelos canais sensoriais. Assim, o sistema nervoso central escolhe a fonte principal para controlar a postura.

Unitermos. Postura, Sistema Nervoso, Órgãos dos Sentidos.

Citaçáo. Kleiner AFR, Schlittler DXC, Sánchez-Arias MDR. O papel dos sistemas visual, vestibular, somatosensorial e auditivo para o controle postural.

\begin{abstract}
The maintenance of a given body orientation is obtained by the complex relation between sensory information and muscle activity. Therefore, this study purpose was to review the role of visual, somatosensory, vestibular and auditory information in the maintenance and control of the posture. Method. a search by papers for the last 24 years was done in the PubMed and CAPES databases. The following keywords were used: postural control, sensory information, vestibular system, visual system, somatosensory system, auditory system and haptic system. Results. the influence of each sensory system and its integration were analyzed for the maintenance and control of the posture. Conclusion. the literature showed that there is information redundancy provided by sensory channels. Thus, the central nervous system chooses the main source for the posture control.
\end{abstract}

Keywords. Posture, Nervous System, Sense Organs.

Citation. Kleiner AFR, Schlittler DXC, Sánchez-Arias MDR. The role of visual, vestibular, somatosensory and auditory systems for the postural control.
Trabalho realizado no Instituto de Biociências da UNESP/RC, no Departamento de Educaçáo Física, Rio Claro-SP, Brasil.

1. Mestre, Biodinâmica da Motricidade Humana, Linha de Pesquisa: Controle e Coordenaçáo de Habilidades Motoras, Unesp, Rio Claro-SP, Brasil. / Doutoranda em Biomecânica, Laboratório de Instrumentação para Biomecânica, Faculdade de Educação Física - FEF da Universidade de Campinas - Unicamp, Campinas, Brasil.

2. Mestre, Biodinâmica da Motricidade Humana, Linha de Pesquisa: Controle e Coordenação de Habilidades Motoras, Unesp, Rio Claro-SP, Brasil. 3. Mestre, Biodinâmica da Motricidade Humana, Linha de Pesquisa: Controle e Coordenação de Habilidades Motoras, Unesp, Rio Claro-SP, Brasil/ Assistente de professor, Faculdade de Fisioterapia da Universidade Del Rosário, Bogotá, Colombia.
Endereço para correspondência: Ana FR Kleiner Avenida Érico Veríssimo, s/n Barão Geraldo, Cidade Universitária "Zeferino Vaz" Caixa Postal 6134, CEP 13083-970, Campinas-SP, Brasil. Tel.: +55 $199783-7808$ E-mail: anafrkleiner@gmail.com 


\section{INTRODUÇÃO}

Controle Postural é por definição a habilidade de um indivíduo assumir e manter a posição desejada durante uma atividade estática ou dinâmica ${ }^{1}$. Desta forma, para um indivíduo se manter estável a habilidade de constantemente controlar o corpo diante a perturbações internas e externas é essencial ${ }^{2}$. Essas perturbaçôes podem ser provenientes de forças da gravidade ou relativas à superfície de apoio dos pés ${ }^{3}$, bem como pela realização de movimentos voluntários do próprio corpo ou partes dele $\mathrm{e}^{4}$.

O controle postural está presente em cada movimento realizado, onde contraçóes musculares apropriadas ocorrem baseadas em informaçōes sensoriais garantindo a posição corporal desejada ${ }^{2,5-7}$. Estas informaçóes sensoriais, provenientes dos sistemas visuais, vestibulares, auditivos e somatossensoriais auxiliam o Sistema Nervoso Central na realização de ajustes posturais. Cada sistema sensorial fornece informaçóes com características únicas, pois cada classe de receptores opera de maneira ótima em frequência e amplitude específicas. Desta forma, o objetivo deste artigo foi discutir o papel das informaçôes sensoriais visuais, vestibulares, auditivas e somatosensoriais, bem como sua integração para manutenção e controle postural.

\section{MÉTODO}

Este artigo é uma revisão de literatura não sistemática e teve como banco de dados o Portal Periódicos CAPES e PUBMED para a busca de artigos publicados nas décadas de 1970, 80, 90 e primeira década do século XXI (datados entre os anos 1973 a 2007). Os conceitos base da área controle motor referidos a estudos de controle postural foram aplicados para a busca dos artigos, desta forma as palavras-chave utilizadas foram: postural control, sensory information, vestibular system, visual system, somatosensory system, auditory system e haptic system. Assim, para este artigo, foram considerados estudos feitos em seres humanos, o nível de reconhecimento dos pesquisadores na área, e estudos clássicos da área de controle postural.

\section{RESULTADOS}

Nos tópicos a seguir, são apontados os principais achados dos artigos encontrados. Em primeira instância, é discutido o papel das informaçóes sensoriais para o equilíbrio postural, em seguida é explorada separadamente a influência de cada sistema sensorial, e sua integração para a manutençẫo e controle postural.

\section{Informaçóes sensoriais e o equilíbrio postural}

Três modalidades sensoriais são responsáveis pela orientação postural: a Propriocepção que é responsável pelo senso de posição e movimento de uma parte do corpo relativa à outra parte do corpo; a Expropriocepção, responsável pela sensação de posição e movimento de uma parte do corpo em relaçẫo ao ambiente; e a Exterocepção, que é responsável por localizar um objeto no ambiente em relação a outro. $\mathrm{O}$ sistema vestibular é puramente exproprioceptivo ${ }^{6}$. O sistema auditivo é exproprio e exteroceptivo. O sistema somatossensorial é exproprioceptivo e proprioceptivo. E o sistema visual é influenciado pela interação destas três modalidades.

É reconhecido que a participação de cada sistema sensorial na orientação e no equilíbrio postural é dependente do tipo de tarefa que está sendo executada e do contexto onde a mesma é realizada ${ }^{2}$. A seguir, a função de cada um destes sistemas no controle postural é discutida.

\section{Sistema Visual}

O sistema visual é considerado, entre os sistemas sensoriais, o mais complexo ${ }^{8,9}$. Seu funcionamento envolve várias estruturas e mecanismos para a obtenção de informaçóes ambientais, que são obtidas através da refração da luz provenientes das superfícies, objetos, plantas, animais e etc. A luz que entra através da córnea é projetada na retina e transformada em sinais elétricos pelos fotorreceptores. Em seguida, é enviada para centros superiores no sistema nervoso central, através do nervo óptico, para ser processada ${ }^{9,10}$.

A importância do sistema visual para o controle postural é principalmente relacionada à estabilização 
da oscilação corporal. Vários estudos demonstram que durante a manutenção da postura ereta estática, a oscilação corporal aumenta até mais que o dobro quando a informação visual não está disponível ${ }^{11}$. Desta forma, essa informação atua como uma fonte de informação sensorial que propicia uma melhora do desempenho do sistema de controle postural.

Entretanto, observa-se que a contribuição do sistema visual para o controle postural não está apenas restrita em manter os olhos abertos, mas também depende das características do estímulo visual. Foi sugerido que o sistema de controle postural estabiliza a oscilação corporal relativa ao ambiente, minimizando a variação do cenário ambiental projetado na retina ${ }^{11}$. Esta hipótese baseou-se no fato de que qualquer deslocamento da imagem projetada na retina indicaria mudança de posição do corpo e, então, seria utilizada para promover correçôes apropriadas. Assim, estas correçóes realizadas de forma contínua, reduziriam a oscilação final.

Assumindo esta proposta de minimização do deslocamento da imagem da retina, alguns estudos ${ }^{11,12}$ destacaram que quanto maior a precisão da imagem na retina, maior é a redução da oscilaçấo corporal decorrente desta informação. Por exemplo, oscilação corporal na posição em pé parado aumenta conforme a distância entre a pessoa e o ponto de fixaçãoo ${ }^{11,12}$. Em distâncias curtas, a projeção do cenário na retina é maximizada e pequenas alterações na posição corporal são percebidas. Por outro lado, para distâncias longas, o oposto acontece.

Entretanto, o efeito da distância no uso da informação visual para o controle postural é apenas um dos aspectos que altera a qualidade da informação visual na retina. Foi verificado que a acuidade visual, o nível de luminosidade e de contraste são aspectos relacionados à informação visual que influenciam o controle postural $^{12}$. Embora a informação visual seja importante para minimizar a oscilação corporal, ela também pode ser utilizada para induzir oscilação corporal.

A influência da visão no controle postural também foi demonstrada através da manipulação das informaçóes visuais através do paradigma da "sala móvel" ${ }^{13}$. Neste estudo, indivíduos na posição em pé e parados, foram colocados dentro de uma "sala móvel", cujas paredes se movimentavam para frente e para trás, fazendo movimentos discretos que eram realizados manualmente por um experimentador ${ }^{13}$. Os movimentos dessa sala produziam deslocamentos das imagens na retina que induziam à percepção de movimentos ilusórios do corpo na direção oposta aos movimentos das paredes da sala. Quando as paredes se moviam para frente, as imagens na retina dos indivíduos eram reduzidas, provocando a sensaçẫo ilusória de deslocamento do corpo para trás. Da mesma forma, quando as paredes da sala eram movimentadas para trás, as imagens nas retinas foram maximizadas, induzindo a percepçáo de um movimento ilusório do corpo para frente. Então, para minimizar e compensar esses deslocamentos ilusórios, os indivíduos oscilavam na mesma direção do estimulo visual. Estes resultados sugeriram que a visão atua como fonte integrante do sistema de controle postural e que diante de conflitos sensoriais, decorrentes de informaçôes ilusórias, a visão domina os canais vestibulares e somatossensoriais ${ }^{14}$. Manipulando a informação visual por meio do paradigma da sala móvel, muitos estudos ${ }^{15-17}$ demonstraram que: quando a informação visual é manipulada, através do movimento da sala móvel, ocorre uma indução de oscilação corporal correspondente ao fluxo óptico.

\section{Sistema Somatossensorial}

O sistema somatossensorial difere dos outros sistemas sensoriais porque seus receptores estão espalhados pelo corpo humano. Estes receptores respondem a diferentes tipos de estímulos como toque, temperatura, posição do corpo e dor. Cabe ao sistema nervoso central interpretar a atividade dos receptores e utilizá-los para gerar percepçóes coerentes com a realidade ${ }^{2,18}$.

Sobre os receptores dos pés, vem sendo demonstrado que estes podem interferir no limiar dos neurônios espinhais, nos quais existe uma interação com as informaçôes vestibulares, visuais e proprioceptivas do pescoço. É também reconhecido, que estes mecanorreceptores são capazes de localizar e detectar pequenas mudanças na pressão da sola dos pés para reagir a altas 
alteraçóes de frequência ${ }^{19}$. Os receptores nos pés, pernas e troncos podem ser criticamente importantes para o controle do corpo, particularmente, sobre condiçóes onde o indivíduo se mantém em contato com uma superfície de larga, rígida e estável ${ }^{2}$.

Em um ponto de vista mecânico, o pé pode ser considerado como uma alavanca que produz um momento para anular o efeito da gravidade na posiçáo estática do corpo. Por exemplo, a força de reação do solo necessária para prevenir uma queda é refletida pela magnitude e pela localização da pressão contra a sola dos pés e seus mecanorreceptores. Desta forma, a informação dos mecanorreceptores dos pés tem propriedades que contribuem significativamente para o controle postural ${ }^{19}$.

A informação sensorial da região plantar tem uma importância moderada para a manutenção da postura ereta ${ }^{20}$. Entretanto, o impacto da perda sensorial da regiáo plantar pode ser aumentado caso existam outros déficits de informação proprioceptiva, como são comuns nos casos das neuropatias periféricas. $\mathrm{Na}$ ausência de informação dos mecanorreceptores, o movimento é prejudicado. As neuropatias periféricas que causam perda da somatosensação que vem dos mecanorreceptores dispostos na pele podem estar associadas pelo sinal de Romberg, onde os pacientes são incapazes de permanecer em pé sem assistência externa (uso de bengalas e andadores, por exemplo), com os pés juntos, e com os olhos fechados ${ }^{21}$. Desta forma, sem a informação das diferenças de pressão plantar (informação sensorial da planta dos pés), os reflexos vestibulares são incapazes de manter a postura. $\mathrm{E}$ a visão não pode substituir a perda da informaçáo de mecanorreceptores ${ }^{21}$.

A informação somatossensorial não informa apenas o sistema nervoso sobre as qualidades da superfície de suporte, mas também sobre a força que o corpo exerce sobre estas superfícies ${ }^{2}$, a posição e a velocidade de todos os segmentos corporais, seu contato com objetos externos, inclusive o chão, e a orientação da gravidade.

A percepção háptica, um dos componentes do sistema somatossensorial, é decorrente de esforços coordenativos táteis-cinestésicos durante atos exploratórios utilizados principalmente na manipulação de objetos com o objetivo de detecçáo de tamanho, formas, texturas, etc ${ }^{22,23}$. No campo da percepção háptica, foi proposto um sistema âncora que pode auxiliar no controle postural através da aquisição da informação do ambiente por sua exploração com ferramentas não-rígidas ${ }^{22}$. $\mathrm{O}$ objetivo de tal uso é o de obter informaçóes do ambiente que possam auxiliar na manutençáo do equilíbrio. A exploração do ambiente por meio de ferramentas (cabos rígidos ou flexíveis) proporciona uma espécie de ancoragem entre o animal e o ambiente ${ }^{22,23}$. Esta ancoragem ocorre a partir do uso das informaçóes contidas no ambiente, que permitem, a partir do contato entre as duas extremidades, uma otimização na busca pela estabilidade na postura ereta ${ }^{22,23}$.

A estratégia de toque suave em uma superfície rígida e estacionária também foi utilizada para investigaçáo do controle postural ${ }^{24}$. Neste estudo, indivíduos adultos na posição em pé tocaram com a ponta do dedo indicador uma superfície rígida e estacionária, posicionada ao lado deles, em duas condiçóes experimentais: toque ilimitado e toque limitado a menos de $1 \mathrm{~N}$ de força (toque suave) ${ }^{24}$. Nas duas situações foi constatada uma redução significante na oscilação corporal, comparada com a situação sem toque. Segundo os autores, no caso da situação de toque ilimitado, a redução da oscilação corporal ocorreu em virtude do suporte mecânico fornecido pela superfície. No entanto, no caso do toque limitado, a força aplicada na superfície era suficiente para fornecer suporte mecânico necessário para tal redução da oscilação corporal, sugerindo que a atenuação da oscilação corporal foi decorrente da utilização de informaçáo sensorial proveniente do toque do dedo na superfície estacionária.

Foi observado que o contato com um objeto de referência foi crucial para estabilizar a oscilação corporal, enquanto a realização de uma tarefa de estabilizar o dedo no espaço não provocou qualquer oscilação corporal $^{25}$. Desta forma, estes achados ${ }^{25}$ confirmaram os resultados $\mathrm{de}^{24} \mathrm{e}$, além disso, sugerem que o contato com uma superfície estacionária fornece um referencial que é utilizado pelo sistema de controle postural para 
estabilizar a postura ereta.

$\mathrm{Na}$ ausência da visão a percepção háptica pode ser utilizada para diminuir a oscilação do corpo ${ }^{26}$. Também vem sendo demonstrado que as dicas hápticas de um toque com a ponta do dedo podem melhorar o controle postural na ausência de visão, mesmo que o contato das forças entre uma superfície estável e o dedo esteja distante por um suporte mecânico ${ }^{24}$.

As dicas sensoriais hápticas são transmitidas pelas vias cutâneas, proprioceptivas e cinetésicas, presumidamente, também fornecem alta resolução espacial nos termos de especificar a posição corporal e mudar a posiçáo do corpo. Por conta disso, as dicas hápticas facilitam a estabilidade postural de indivíduos com labyrinthine-defective bem como com pessoas com cegueira congênita ${ }^{27}$, indicando que a informação háptica pode substituir a falta de informação vestibular ou visual de referências espaciais, e pode contribuir, de forma importante, na manutenção do controle postural.

\section{Sistema Vestibular}

Os receptores vestibulares nos canais semicirculares e otólitos maculares são sensíveis à aceleração angular e linear da cabeça, respectivamente. $\mathrm{O}$ sinal do otólito é uma combinação de todas as aceleraçóes lineares agindo sobre a cabeça, incluindo a aceleração constante da gravidade. Assim, os otólitos são estimulados com os movimentos da cabeça com respeito à gravidade. Porém os sinais dos otólitos sozinhos não são provavelmente responsáveis para o nosso sentido de "verticalidade"2.

Os canais semicirculares, como acelerômetros angulares, são sensíveis a altas frequências de movimentos da cabeça, assim como os otólitos. Os canais anteriores e posteriores, os quais detectam as posiçóes pitch e roll são especialmente importantes para detectar a oscilação postural que ocorre com a rápida flexão ou extensão do quadril, mas não para detectar a baixa frequência de oscilação em pé2.

A estimulação galvânica foi aplicada sobre o aparelho vestibular em indivíduos normais em postura ereta sobre uma plataforma móvel ${ }^{28}$. Os resultados aponta- ram que quando o estímulo ocorreu com o movimento da base de suporte, a resposta postural foi baseada em uma interação de ambas as informaçóes (vestibular e somatossensorial). Quando estes estímulos foram apresentados com considerável atraso, as informaçôes foram tratadas sem nenhuma interação. Assim, foi concluído que a contribuição da informação vestibular acontece principalmente no final de uma tarefa de equilíbrio dinâmico ${ }^{28}$.

Em outro estudo com estimulação galvânica ${ }^{29}$, reforçando a importância do sistema vestibular em condiçôes de déficit de informação visual e somatossensorial, foi observado que os reflexos vestíbulo-espinhais foram maiores quando os participantes permaneciam em uma base de suporte estreita, em superfície macia, e desprovidos de visão e apoios externos.

A ausência ou a falha da informação vestibular pode alterar o controle da postura. Para manter o controle da postura ereta, o controle postural seleciona estratégias apropriadas. Duas das estratégias posturais mais estudadas são a estratégia do tornozelo e a estratégia do quadril ${ }^{30}$, que se diferenciam, entre outros aspectos, pelo grupo muscular recrutado durante uma instabilidade postural no plano sagital. A ausência da informação vestibular resulta na ausência da estratégia do quadril e sugere que a informação vestibular é necessária quando a tarefa de equilíbrio dinâmico requer o uso da estratégia do quadril ${ }^{31}$. Também observam que na ausência de informação somatossensorial, o ajuste postural compensatório baseia-se na estratégia do quadril. No entanto, a ausência de um desses sistemas é suficiente para atrasar ou desorganizar a resposta postural. Pode-se dizer que o sistema vestibular orienta as informaçóes geradas pelos movimentos da cabeça durante as posturas estáticas e dinâmicas do corpo diante da gravidade.

\section{Sistema Auditivo}

Prejuízos na audição e no equilíbrio, bem como as quedas, são comuns entre as pessoas idosas ${ }^{32,33}$, e estes, podem se correlacionar, primeiro, porque a audição fornece informação acústica sobre o ambiente, nos 
habilitando a notar e evitar irregularidades ambientais que possam provocar quedas. Segundo, a estrutura e função do ouvido interno sugerem que tanto o ouvido quanto os órgãos vestibulares podem compartilhar fatores etiológicos em comum. $\mathrm{O}$ ouvido e os órgãos vestibulares são anatomicamente localizados próximos uns dos outros, compartilham a circulação sanguínea, são inervados pelo $8^{\circ}$ nervo craniano, e têm mecanorreceptores sensoriais, os quais detectam o som, movimentos da cabeça e orientação espacial. A perda destas células receptoras pode ocorrer na cóclea ${ }^{34}$ e no órgão vestibular ${ }^{35}$ durante o processo de envelhecimento, mas também através de uma variedade de lesóes, incluindo exposição a drogas ototóxicas e barulho ${ }^{36}$.

Estudos com associaçóes entre acuidade auditiva e equilíbrio postural, bem como acuidade auditiva e quedas, são escassos, e os resultados apresentados são contraditórios. Alguns estudos mostram correlação entre maior exposição ao barulho e prejuízos no controle postural ${ }^{37,38}$. Algumas evidências apontam que os acidentes domésticos, em sua maioria quedas, são mais comuns em pessoas com problemas auditivos do que com pessoas $\mathrm{sem}^{39}$. Entretanto, outros estudos encontraram uma pequena ou nenhuma associação entre acuidade visual e equilíbrio postural ou quedas ${ }^{40-42}$.

A adequação da informação auditiva para o controle da estabilidade postural também pode ser importante em pessoas portadoras de necessidades especiais, em especial, com cegueira congênita. Estas tipicamente tendem a utilizar a informação auditiva para ganhar conhecimento espacial, que em indivíduos com visão normal é garantida pela visão ${ }^{43}$. Por exemplo, quando se locomovem pela rua, pessoas cegas utilizam o som do tráfego para o alinhamento paralelo ou perpendicular ${ }^{44}$ ou para se aproximar de um veículo motorizado ${ }^{45}$. Pois, na falta das informaçóes visuais típicas, áreas não visuais de percepção tornam-se mais desenvolvidas em pessoas cegas $^{46}$, auxiliando-as a se locomover com eficiência no ambiente.

Desta forma, pouco ainda se sabe sobre as contribuiçóes do sistema auditivo no controle postural. Entretanto, pode-se afirmar que o sistema auditivo é de suma importância para a orientação espacial, em especial, para portadores de deficiência visual.

\section{Integraçáo e a repesagem das informaçóes sensoriais para o controle postural}

O controle postural é o controle dos arranjos dos segmentos corporais baseado em informaçóes sensoriais de diferentes fontes. Estas informaçóes permitem formar uma representação interna do ambiente, representando e reconhecendo a posição do movimento de cada parte do corpo. Como discutido, o controle postural utiliza informaçóes dos sistemas sensoriais. Observa-se que a abundância de informações é um fato que garante a estabilidade postural mesmo na deficiência de um sistema. Acredita-se que o nível das informaçóes sensoriais seja modulável e redundante ${ }^{8}$. A modulação de informação sensorial depende de estados de atenção e mesmo da integridade de cada um dos sistemas sensoriais ${ }^{47}$.

A separação anatômica dos sistemas sensoriais envolvidos com o controle postural e a degradaçáo significativa das informaçôes sensoriais, quando fechamos os olhos ou permanecemos em superfícies moles ou macias, sugerem que o sistema nervoso tem a habilidade de mudar discretamente a fonte principal de informação sensorial ${ }^{8}$.

A transição de uma fonte sensorial para outra é ajustada por uma simples dica sensorial, escolhida em uma lista limitada de opçóes, em vez da combinação de dicas sensoriais ou sobre aspectos gerais do ambiente. A transição de fontes sensórias corresponde, por exemplo, a passar da dominância da informação visual para a somatossensorial ${ }^{48}$. Logo, o sistema nervoso escolhe a fonte principal para controlar a postura ${ }^{8}$, e quando a transição de uma fonte de percepção para outra é realizada, acontece de forma abrupta, assim usa uma informaçáo sensorial de cada vez. A dominância de um sistema sensorial sobre o outro é uma estratégia do sistema nervoso evitar conflitos de informaçóes ${ }^{49}$.

Além disso, é importante considerar o contexto em que a tarefa está sendo realizada ${ }^{50}$, pois é em função deste que o sistema de controle postural se baseia mais em uma informação sensorial do que em outras ${ }^{51}$. Se- 
gundo esses autores o peso atribuído a cada canal sensorial depende do quão útil a informação fornecida por este é, para que o sistema de controle postural alcance o seu objetivo. Assim, dependendo da tarefa, uma informação sensorial pode tornar-se mais preponderante do que outras, porém, em outra situação, esta preponderância pode ser alterada ou até mesmo invertida.

A informação sensorial influencia a ação motora ao mesmo tempo em que a ação motora altera o fluxo de informaçáo sensorial, com o objetivo de atingir ou manter o equilíbrio e a orientação postural, denominado ciclo percepção-ação ${ }^{52}$. Para testar o acoplamento entre percepção-ação, foi usado o paradigma da sala móvel, que inicialmente verificava a influência das alteraçóes do fluxo óptico no controle postural, ocasionado por movimentos do cenário visual, essas alteraçóes eram ajustes coerentes na mesma direção do estímulo visual ${ }^{13}$. A maioria dos estudos com a sala móvel verificava o papel do sistema visual no controle postural, em variadas populaçôes e faixas etárias.

Atualmente, esse paradigma passou a ser utilizado para verificar o relacionamento entre informação sensorial e ação motora, onde os parâmetros utilizados pelo sistema podem mudar em função da característica da informação visual, modificando sua frequência natural de oscilação, indicando a ocorrência de adaptação do sistema de controle postural ${ }^{53}$.

Em estudos recentes, o peso dado a cada modalidade sensorial foi investigado. A repesagem sensorial é uma informação que contribui para a flexibilidade e estabilidade do controle da postura em pé, e caracterizado por uma náo-linearidade do sistema de controle postural $^{54,55}$.

Quando ocorre uma perda completa de um sistema sensorial, outro sistema deve compensar essa perda, alterando a capacidade de resposta desse sistema, ou seja, o sistema aumenta o peso de uma modalidade sensorial enquanto diminui o peso das outras, e essa repesagem sensorial é vista como uma variável dinâmica que depende da dimensão do estímulo do movimento $^{55}$, e tem caráter contexto-dependente ${ }^{56}$. A repesagem sensorial em crianças pode ser visto desde os 4 anos de idade, assim como o desenvolvimento de repesagens simultâneas em duas modalidades sensoriais. Isso é importante no processo do desenvolvimento, pois serve de fundação para os comportamentos funcionais de alcance e de locomoção ${ }^{54}$.

\section{CONCLUSÃO}

Este texto destacou a importância de cada sistema sensorial no controle postural, através de estudos que analisaram essa relação complexa e dinâmica pela manipulação da informação sensorial proveniente de um ou mais canais sensoriais e sua influência no sistema de controle postural.

Não há controle postural sem que haja integração das informaçóes sensoriais, pois existe uma forte relação entre informação sensorial e ação motora. A entrada de informação sensorial influencia a realização das atividades motoras e ao mesmo tempo, a realização de uma ação motora influencia o modo como a informação sensorial é obtida. A integraçáo de todas as modalidades sensoriais fornece algumas vezes conflito ao sistema, ou seja, o sistema não sabe ao certo qual informação é mais coerente, dando pesos diferentes para cada uma delas. $\mathrm{Na}$ falta de uma informação, outro sistema pode aumentar sua capacidade de perceber o movimento do corpo ou o movimento do ambiente.

E por fim, as bases do controle postural estão relacionadas com vários fatores, o primeiro deles é o funcionamento do sistema nervoso central em harmonia com os outros sistemas, pois é ele que processa as informaçóes sensoriais e envia comandos para as respostas musculares. As respostas musculares são dependentes da grandeza do estímulo, quando o estímulo é pequeno a ativaçáa muscular ocorre no nível do tornozelo, quando a perturbação é maior, a ativação pode ser ao nível do quadril ou ainda uma reaçáo mista, assim como as reaçóes antecipatórias e reativas, que dependem do bom funcionamento do SNC e suas vias para o controle da postura e a orientação no espaço. As características do estímulo definem a melhor estratégia a ser utilizada para o controle da postura e quais as informaçôes e modalidades sensoriais que terão mais 
relevância. Com tudo, um dos maiores desafios da área de comportamento motor é entender como ocorre o relacionamento entre informação sensorial e o controle dos movimentos.

\section{REFERÊNCIAS}

1.Cupps B. Postural control: a current review. Neuro Developmental Treatment 1997,1:3-8.

2.Horak FB, MacPherson JM. Postural orientation and equilibrium. In: Rowell L, Shepard J (eds). Handbook of physiology. New York: Oxford, 1996, p.255-92.

3.Latash ML. Neurophysiological basis of movement. Champaign: Human Kinetics, 1998, p.163-78.

4.Cordo PJ, Nashner LM. Properties of postural adjustments associated with rapid arm movements. J Neurophysiol 1982;47:287-308.

5.Winter DA. Kinetics: forces and moments of force, biomechanics and motor control of human movement. Waterloo: Wiley, Interscience, 1990, p.74-102.

6.Nashner LM. Analysis of stance posture in humans. In: Towe AL, Lusche ES. Handbook of behavior neurology. New York: Plenum Press, 1981, v.5, p. 527-65.

7.Ghez C. Posture. In: Kandel ER, Schwortz JH, Jessell A. Principles of neuroscience. 3rd ed, New York: Elsevier, 1991, p.596-607.

8.McCollum G, Shupert CL, Nashner LM. Organizing sensory information for postural control in altered sensory environments. J Theorical Biol, 1996;180:257-70

http://dx.doi.org/10.1006/jtbi.1996.0101

9.Manson C, Kandel ER. Central Visual Pathways. In: Kandel ER, Schwortz JH, Jessell A. Principles of neuroscience. 3rd ed. New Yourk: Elsevier, 1991, p.420-39.

10.Kandel ER. Perception of motion, depth, and form. In: Kandel ER, Schwortz JH, Jessell A. Principles of neuroscience. 3rd ed. New York: Elsevier, 1991, p.440-65.

11.Paulus M, Traube A, Brandt T. Visual stabilization of posture physiological stimulus characteristics and clinical aspects. Brain, 1984;107:1143-63. http://dx.doi.org/10.1093/brain/107.4.1143

12.Paulus W, Straube A, Krafczyk S, Brandt T. Differential effects of retinal target displacement changing disparity in the control of anterior/posterior and lateral body sway. Experimental Brain Research, 1989;78:243-52.

http://dx.doi.org/10.1007/BF00228896

13.Lishman JR, Lee DN. The autonomy of visual kinaesthesis. Perception, $1973 ; 2: 287-94$.

http://dx.doi.org/10.1068/p020287

14.Lee DN, Lishman JR. Visual proprioceptive control of stance. J Human Movement Studies, 1975;1:87-95.

15.Freitas Júnior PB, Barela JA. Postural control as function of self- and object-motion perception. Neuroscience Letters, 2004;369:64-8.

http://dx.doi.org/10.1016/j.neulet.2004.07.075

16.Polastri PF, Barela JA. Perception-Action coupling in infants with down syndrome: effects of experience and practice. Adapted Physical Activity Quartely, 2005;22:39-56.
17.Prioli AC, Freitas Júnior PB, Barela JA. Physical Activity and Postural Control in the Elderly: coupling between visual information and body sway. Gereontology, 2005;51:145-8.

http://dx.doi.org/10.1159/000083984

18. Martin JH, Jessell TM. Anatomt of the Somatic Sensory System. In: Kandel ER, Schwortz JH, Jessell A. Principles of neuroscience. 3rd ed. New Yourk: Elsevier, 1991, p.596-607.

19.Magnusson M, Enbom R, Johansson R, Pyykko I. The importance of somatosensory information from the feet in postural control in man. In: Brandt I, Paulus W, Bles W, Dieterich R, Krafeyzk S, Strambe A. Disorders of posture and gait. Stuttgart: Georg. 1990, p.194-7.

20.Meyer PF, Oddsson LI, De Lucca CJ. The role of plantar cutaneous sensation in unperturbed stance. Experimental Brain Research, 2004;154:505-12.

21.Rothwell J. Control of human voluntary movement. $2^{\text {a }}$. ed. London: Chapmann \& Hall, 1994, 520p.

22.Castro EM. Developing an "anchor" system to enhance postural control. Motor Control, 2004;8:339-58.

23.Castro EM. Sensibilidade háptica no controle postural através do uso de um sistema âncora. Resultados Preliminares. In: III Seminário de Comportamento Motor, Gramado. Anais do III Seminário de Comportamento Motor, 2002;1:13.

24.Jeka JJ, Lackner JR. Fingertip contact influences human postural control. Experimental Brain Research, 1994;100:495-502.

http://dx.doi.org/10.1007/BF02738408

25.Krishnamoorthy V, Slijper H, Latash ML. Effects of different types of light touch on postural sway. Experimental Brain Research, 2002;147:71-9. http://dx.doi.org/10.1007/s00221-002-1206-6

26.Riley MA, Stoffregen TA, Grocki MJ, Turvey MT. Postural stabilization for the control of touching. Human Movement Science, 1999;18:795-817. http://dx.doi.org/10.1016/S0167-9457(99)00041-X

27.Jeka JJ, Easton RD, Bentzen BL, Lackner JR. Haptic cues for orientation and postural control in sighted and blind individuals. Percept Psychophys, 1996;58:409-23.

http://dx.doi.org/10.3758/BF03206817

28.Hlavacka F, Shupert C, Horak FB. The timing of galvanic vestibular stimulation affects responses to plataform translation. Experimental Brain Research, 1999;821:8-16.

29. Welgampola MS, Colebatch JG. Vestibuloespinal reflexes: quantitative effects of sensory feedback and postural task. Experimental Brains Research, 2002, 139, 345-353.

http://dx.doi.org/10.1007/s002210100754

30.Nashner LM, McCollum G. The organization of postural movements: a formal basis and experimental synthesis. Behavior and Brain Sciences, 1985;8:135-72.

http://dx.doi.org/10.1017/S0140525X00020008

31.Horak FB, Nashner LM, Diener HC. Postural strategies associated with somatosensory and vestibular loss. Experimental Brain Research, 1990;82:167-77.

http://dx.doi.org/10.1007/BF00230848

32.Kannus P, Sievänen H, Palvanen M, Järvinen T, Parkkari J. Prevenction of falls and consequent injuries in elderly people. Lancet, 2005;366:1885-93. http://dx.doi.org/10.1016/S0140-6736(05)67604-0

33.Uimonen S, Huttunen K, Jounio-Ervasti K, Sorri M. Do we know the real need for hearing rehabilitation at the population level? Hearing impairments in the 5- to 75-year-old cross-sectional finish population. British Journal of Audiology, 1999;33:53-59. http://dx.doi.org/10.3109/03005364000000099 
34.Schurnecht HF, Gacek MR. Cochlear pathology in presbycusis. Annals of Otology, Rhinology and Laryngology, 1993;102:1-16.

35.Rosenhall U. Degenerative patterns in the aging human vestibular neuro-epithelia. Acta Oto-Laryngologica, 1973;76:208-20.

http://dx.doi.org/10.3109/00016487309121501

36.Selimogll E. Aminoglycoside-induced ototoxicity. Current Pharmaceutical Design, 2007;13:119-26.

http://dx.doi.org/10.2174/138161207779313731

37.Juntunen J, Matikainen E, Ylikoski J, Ylikoski M, Ojala M, Vaheri E. Postural body sway and exposure to hight-energy impulse noise. Lancet, 1987;2:261-4.

http://dx.doi.org/10.1016/S0140-6736(87)90840-3

38. Kilburn KH, Warshaw RH, Hanscom B. Are hearing loss and balance dysfunction linked in construction iron workers? British J Industrial Med, 1992;49:138-41.

39.Euci ED, Ergin F, Beser E. Home accidents in the elderly in Turkey. The Tohoku J Experimental Med, 2006;209:291-301. http://dx.doi.org/10.1620/tjem.209.291

40.Enrietto JA, Jaconson KM, Boloh RN. Aging effects on auditory and vestibular responses: a longitudinal study. American J Otolaryngol, 1999;20:371-8.

http://dx.doi.org/10.1016/S0196-0709(99)90076-5

41.Era P, Schroll M, Yttin H, Gause-Nilsson J, Heikkinen E, Steen B. Postural balance and its sensory-motor correlates in 75-year-old men and women: a cross-national comparative study. Journals of Gerontology Series A: Biological Sciences and Medical Sciences, 1996;5:M53-63.

42.Purchase-Helzener EL, Cauley JA, Faulker KA. Hearing sensitivity and the risk of incident falls and fractures in older women: the study of osteoporotic fractures. Annals of Epidemiology, 2004;14:311-8.

http://dx.doi.org/10.1016/j.annepidem.2003.09.008

43.Asmead DH, Davis DL, Northington A. The contribution of listeners' approaching motion to auditory distance perception. Journal of Experimental Psychology: Human Perception and Performance, 1995;21:239-56. http://dx.doi.org/10.1037/0096-1523.21.2.239

44.Hill EW, Ponder P. Orientation and mobility techniques: a guide for practitioner. New York: American Foundation for the Blind, 1976, 119p. 45.Schiff W, Oldak R. Accuracy of judging time to arrival: effects of modality, trajectory, and gender. Journal of Experimental Psychology: Human
Perception and Performance, 1990;21:303-16.

http://dx.doi.org/10.1037/0096-1523.16.2.303

46.Rauschecher JP. Compensatory plasticity and sensory substitution in cerebral cortex. Trends in Neuroscience, 1995;6:203-5.

47.Day BL, Cole J. Vestibular-evoked postural responsesin the absence of somatosensory information. Brain, 2002;125:2081-8.

http://dx.doi.org/10.1093/brain/awf212

48.Maurer C, Mergner T, Bolha B, Hlavacka F. Vestibular, visual and somatosensory contributions to human control of upright stance. Neuroscience Letters, 2000;281:99-102.

http://dx.doi.org/10.1016/S0304-3940(00)00814-4

49.Massion J, Woollacott MH. Posture Control. In: Bronstein AM, Brandt T, Woollacott MH. Clinical disorders of balance, posture and gait. London: Arnold, 1996, 480p.

50.Streepey JW, Ângulo-Kinzler RM. The role of task difficulty in the control of dynamic balance in children and adults. Human Movement Science, 2002;21:423-38.

http://dx.doi.org/10.1016/S0167-9457(02)00104-5

51.Jeka JJ, Oie KS, Kiemel T. Multisensory information for human postural control: integrating touch and vision. Experimental Brain Research, 2000;134:107-25.

http://dx.doi.org/10.1007/s002210000412

52.Barela JA. Estratégias de controle em movimentos complexos: Ciclo percepção-ação no controle postural. Rev Paul Educ Fís, 2000;1(supl.3):79-88. 53.Dijkstra TMH, Gielen CCAM, Melis BJM. Postural responses to stationary and moving scenes as a function of distance to the scene. Human Movement Science, 1992;11:195-203.

http://dx.doi.org/10.1016/0167-9457(92)90060-O

54.Bair WN, Kiemel T, Jeka JJ, Clark JE. Development of multisensory reweighting for posture control in children. Exp Brain Res 2007;183:435-46. http://dx.doi.org/10.1007/s00221-007-1057-2

55.Oie KS, Kiemel T, Jeka JJ. Multisensory fusion: simultaneous re-weighting of vision and touch for the control of human posture. Cognition Brain Research, 2002;14:164-76.

http://dx.doi.org/10.1016/S0926-6410(02)00071-X

56.Forssberg H, Nashner LM. Ontogenetic development of postural control in man: adaptation to altered support and visual conditions during stance. The J Neuroscience, 1982;2:545-52. 